\title{
A Quantitative Treatment to Data from Computer-Supported Collaboration: An Ontological Approach
}

\author{
Luis Casillas \\ University of Guadalajara, Department of \\ Computer Sciences. Av. Revolucion 1500, \\ 44840 Guadalajara, Mexico \\ luis.casillas@red.cucei.udg.mx
}

\begin{abstract}
Collaborative activity performed over specific platforms, designed for such purpose, can provide a deep knowledge about the roles, intentions and effects regarding participants and their interaction among themselves and with the knowledge objects available. This study aims at proposing a structured process for gathering the semantics of the activity hidden behind the raw data collected in log files from CSCL platforms. The proposal is based on exploring the semantic elements (activity awareness) through a social networks analysis (SNA). The main focus of our work is to match different behavioral profiles detected in the collaborative activity from CSCL with the formal profiles identified inside a complex concept-network. This network defines an ontology that describes the behavior expected when collaborating in different scenarios and types of activity. When a certain activity sequence matches with a predefined pattern, the concepts related to the pattern are then bound to the real activity sequence.
\end{abstract}

\section{Introduction}

The analysis of collaborative activity in virtual environments has become an important task when supervising and monitoring the performance of learning groups. Regular face-to-face collaborative interaction includes a diversity of communication elements beyond the spoken language. This complex message exchange would imply, hopefully, a synchronization of minds, which supports a shared network of concepts. The experience of being present in situ when collaborating provides a complete feeling of situation-awareness [4]. Collaboration in virtual environments does not provide this rich experience of having situation-awareness. Limited communication channels restrict the full transfer of the messages generated by each member of the workgroup.

\author{
Thanasis Daradoumis \\ Open University of Catalonia, Department \\ of Computer Sciences. Rambla Poblenou \\ 156, 08018 Barcelona, Spain \\ adaradoumis@uoc.edu
}

Collaboration analysis is then oriented to discover the intentions and effects of every primitive collaborative action [1]. It is evident that mutual understanding among peers is the first step to solve any problem that may appear when different people are involved in the search of a solution. Every member of the group might have a different approach of the problem and its possible solution. Though collaboration is not a formal and ordered process, the initial stages must be clearly oriented to model a common structure of concepts regarding the problem and its solution. Our work is oriented to discover profiles of behavior shown in CSCL platforms.

Discovering the knowledge hidden among the big volume of data stored in virtual environments supporting collaboration is the main task that should be performed during collaboration analysis. Hence, the specialists performing the analysis must collect and process data through mining techniques and do the required inference for filling up those indicators defined for measuring the collaboration success. Along this work, we will propose an ontological approach for detecting the behavioral profiles from primitive sequences of activity.

\section{Related work}

The analysis of the collaborative activity in Computer Supported Collaborative Learning (CSCL) environments implies a significant effort as concerns the complexity of gathering knowledge from the raw data stored by these platforms. In particular, the work performed in [9] has been oriented to carry out an analysis from data coming from a networked collaborative environment to study the interaction which is organized in a temporal structure. Hence, it was possible to compare current behaviors by matching them with expected behaviors.

Another interesting approach for performing collaborative analysis is presented in [8], which presents a Model of Effective Collaborative Learning 
as a framework that provides meaning to collaboration acts by classifying the primitive actions into skills, sub-skills and attributes. This model depends on the use of certain "sentence openers" that express the intentions of the collaborators; however, this restricts the flexibility of collaboration due to the mechanization of communication. Nevertheless, it is clearly understandable that the complexity of free-style human communication is an important challenge for current computational approaches. This trend of collaboration analysis has been maintained in subsequent research work of [6] and [7], focusing on the search for certain messaging structures.

Using models for analyzing collaboration is becoming a key aspect. A model gives coherence to the study of collaboration phenomena by classifying primitive acts in predefined slots, and gathering semantics in the process. The study developed by [2] deals with the collaboration analysis through a layered model, which breaks down the analysis goals in different levels. The lower level is related to raw events generated by a CSCL tool, whereas the higher level is related to abstract collaborative analysis categories. The problem with this approach is the complexity that presents when trying to infer abstract behaviors from the very raw data collected in log files from CSCL tools. Nevertheless, collaborative analysis under such perspective is more flexible, though flexibility is always harder to implement.

Another approach for performing the analysis of collaboration in presented in the study of [3], which copes with the problem from a neural networks perspective. Log files are consumed through a mechanism that classifies information using a sui generis neural net. This approach can offer useful information in-between the structure of the neural network and not only in the output layer (as usually happened with conventional neural networks). Every element in this neural net has information regarding an indicator of collaboration activity, while at the same time the indicator is feeding some neurons in the next layer; this process prepares the indicators of the following layer.

The current proposal aims at performing a collaboration analysis in two stages. The first one deals with collecting and processing raw data from a CSCL platform, and applies a quantitative analysis method to fill up a series of indicators. This stage will be subsequently completed with an approach that will allow us to analyze conversations in order to discover the intentions and effects of certain behaviors (this will constitute an improvement to the approach suggested by Soller). This second stage is currently under development and will provide an automatic conversation analysis. This paper focuses on the development of an ontological approach that completely covers the first stage of the analysis.

\section{Collaboration Analysis}

The core of our task is to develop a precise and useful approach for the analysis of the collaborative interactions of participants in a CSCL environment, involving different analysis dimensions. According to the experience acquired through the review of several cases, we identified different strategies to face the collaboration analysis that go from the simple collection of data and results in spreadsheets, queries to data bases, data mining and decision trees, up to the use of agents with specifications that satisfy different criteria for measuring the collaborative activity. Naturally, simple approaches are easy to design and implement, but their results are usually weak and lack some way of automatic interpretation. Developing a more advanced solution implies an extra effort, but the benefits gained are manifold, since we can obtain a variety of results both in depth and quality, which provides a more powerful tool for decision making and interaction monitoring.

One may initially consider that the complexity of performing a collaboration analysis in online human interaction through a computational solution could be apparently low due to the restrictions of the communication channels available, which reduces the amount of information elements produced. Indeed, the restrictions in transferring information could falsely imply that a rather simple solution could suffice; however, it is just the absence of certain information elements which is the cause of complexity, due to the need to fill some important gaps. These gaps refer to certain messaging elements that complement the regular communication pieces that constitute the whole human communication system, such as non-verbal messaging. Some of these non-verbal communication elements could help understand several collaborative issues; therefore special efforts must be made to discover some of the participants' interests and intentions lying beneath their collaboration acts.

Taking these considerations into account, we first need to carry out a preliminary process so that we can understand the way collaborative interaction evolves and then build a method for gathering higher-level semantics indicators from primitive collaborative actions. To this end, we developed temporal schemata capable to work as an abstract framework for modeling the chronological phenomena that lie behind the collaborative activity, based on the temporal-logics 
tools proposed in [5]. These temporal axioms are the main axis regarding the understanding of the collaboration analyzed. Our study and perspective about collaboration has two dimensions. The first one is related to the analysis of tacit collaborative actions which result from the simple circuit of putting knowledge objects in shared spaces which are then being accessed by other peers who have the required permission to participate in that shared space. The second dimension is more oriented to the conversation analysis performed on online interactions among participants. This second stage is now under development. Although this paper does not include results from that part of the study, we just mention it since our approach aims at providing an integrated solution to the problem of collaborative interaction analysis and awareness. To this end, we show some important results from the first stage of our study.

Our approach has been applied in real collaborative learning situations that took place in the Basic Support for Cooperative Work (BSCW) platform (http://bscw.uoc.es/). BSCW is able to record many types of primitive actions (interactions, transactions, accesses, etc.) performed by users in log files. These data provide an important source of information in order to study both the individual and group performance in learning. In fact, there are thousands of data entries recorded from the interactions among users; more than 25 megabytes per semester. The core of our quantitative-analysis stage consists in processing the whole data stored in log files to build a social network based on the automatic-detection of interactions according to our collaboration model.

The way of collecting and organizing the information regarding the transactions made inside BSCW provides important elements for building a social network. This network represents the users and the knowledge objects stored in the shared workspaces by binding users (students) to those knowledge objects that they access. Students are organized in learning groups, which is an abstract social network. Our aim is to discover and analyze the interactions among these students using different social network analysis techniques.

Since students may belong to more than one teams and thus have access to a variety of shared knowledge objects, the social network developed for carrying out the quantitative analysis stage is not limited to isolated learning teams. Instead, we faced the challenge to build and manage a huge social network that functions as a knowledge server and provider. In fact, the network itself represents a semantic model of the collaboration that takes place among the whole class of participating users. Figure 1 presents a simplified schema of the process used for gathering significant knowledge from the raw data stored in log files, which is a sub-process of the quantitative approach employed. As a matter of fact, the collaborative learning experience held in BSCW produced a huge amount of raw data.

These data were processed through mining techniques in order to discover relations and by means of a model that handles the involved elements via multiple queries and coupling operations over the database. Doing so, we achieve to relate users based on their accesses to knowledge objects.

Once the log files are processed, the information

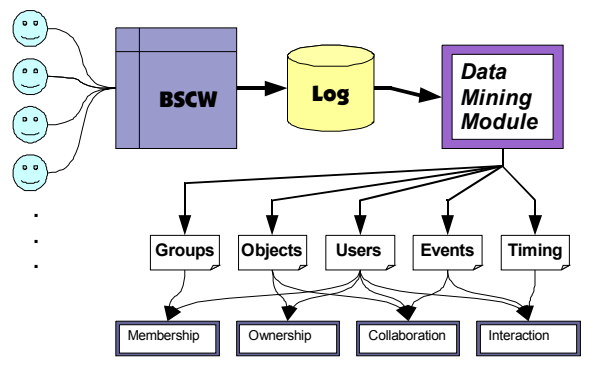

Figure 1. A schema for processing collaborative interaction log files.

regarding collaboration is used for feeding the Knowledge Server, a module which first builds the social network that represents the collaborative activity among users and then it exploits the social network data to model a variety of interaction phenomena a posteriori. It is possible to figure out the functionality of this module by reviewing the schema shown in Figure 2.

In particular, the Knowledge Server becomes the

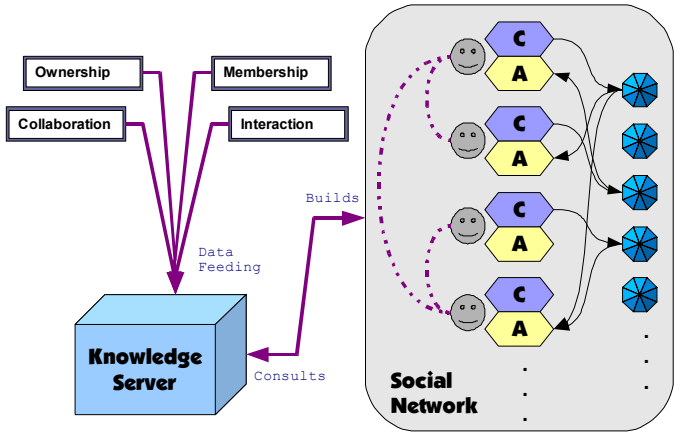

Figure 2. The Knowledge Server module is fed by the coupled model built from activity logs. In the social network, "C" stands for "creates" and "A" for "access".

data provider for the agents which are further employed to fulfill the activity indicators that explain different aspects and issues of collaboration according to specifications defined in an ontology that is 
specifically designed to support our approach of collaboration analysis. The following section aims at explaining this ontology in more detail.

\section{An ontology for describing collaborative activity}

Our ontology constitutes a natural extension of an ongoing effort to provide a rich representation scheme that supports collaboration analysis, which started from the proposal of [8] and went through the work suggested by [2]. As shown in Figure 3, a hierarchical model is built from the general collaborative activity entity which branches to five principal activity indicators: active learning, perception, support, planning and task development, and conflict management. Each branch is subsequently divided into specific aspects of collaboration.

Active learning (AL) refers to the dynamic and adaptable capability for managing the knowledge that is produced during the elaboration of the assigned tasks. The Perception (P) indicator is related to the knowledge flow from one participant to another, as well as to the capability of the receptors not only to acquire new knowledge but also to evaluate its quality and effectiveness towards the accomplishment of the common goals. The Support (S) indicator is used to model the members' actions that aim to provide specific help services and social support at individual and group level. The Planning and Task Development (PTD) dimension refers to the self- and innerorganization of group activity, according to a proposed plan or a set of tasks to be developed. The Conflict Management (CM) indicator deals with the members' ability to handle the differences of opinion that may exist inside a group, through being proactive, able to take decisions and have a constructive critical attitude. Finally, every indicator is further refined into a simpler primitive representation, which is ultimately associated to the primitive actions resulting from the collaborative actions carried out by a member. Figure 3 shows the upper levels of the hierarchical ontology schema that is used to describe the collaborative activity.

This ontology is not directly connected to any specific CSCL tool; therefore an additional mechanism was needed to be inserted between the log-files database and the knowledge server. This inner mechanism is implemented using extractiontransformation-load (ETL) techniques, which unify, contract or expand the stored data in order to achieve the required format that matches them with the ontology.

\section{Implementation of the model}

Based on our analysis goals, the quantitative analysis process was built as an automated mechanism which is fed with raw data to produce a social network. Through its structure, this network shows the semantics of the collaborative activity, by collecting knowledge within its nodes and its edges, as well as by clustering participants around knowledge objects.

Problem complexity was broken down to gather the main system elements. Data were originally composed by means of primitive events from the CSCL tool. Through mining techniques, the data were translated into primitive interaction acts, as explained in Figure 1. Finally interaction acts were processed to produce the collaboration network which models the whole collaborative activity a posteriori. Reaching at this point, it is possible to calculate the different ontology indicators.

Using the object oriented paradigm, we developed a

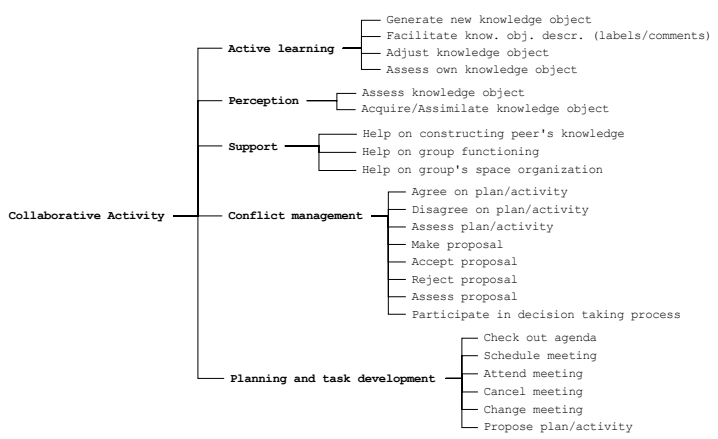

Figure 3. Proposed ontology designed to support the collaboration analysis.

system which builds and manages the social network that supports our knowledge server.

A further important feature and contribution of our approach is the incorporation of two agents to support the Search Module. Due to the multiple redundancies which are present in the primitive interaction acts file, which is a natural consequence of the different accesses to objects along the time, it is necessary to handle the multiple references to the same user, group or knowledge-object, thus avoiding multiple insertions of such elements to the social network. To do so, we employ two agents that take charge of managing the specific names recovery from alternative files; these agents can ran in different computers, decreasing the demand of computer resources. The network has to be constructed only the first time the data are loaded from the $\log$ files. This is possible because of the type of collaborative analysis we are performing; the analysis 
is carried out a posteriori, that is, after a collaborative work and learning phase is finished.

\section{Results and discussion}

Once the network is loaded, the knowledge server is able to respond to queries. Table 1 shows the profile of the global activity discovered throughout the awareness information collected by the Knowledge Server, using the data collected from the BSCW platform during the realization of a real collaborative learning practice that was carried out in our university during the spring 2003 semester.

By reviewing the specific indicators that make up the global activity, shown in table 1, we noted the existence of significant trends which can not be disregarded. In particular, the global activity follows a standard distribution; the most common action performed by users is ReadEvent with a frequency of $74 \%$, followed by CreateEvent with $18 \%$. Besides, object creation spans to almost a $50 \%$ for both Create Document and Create Note actions. Finally, access to objects represents almost a $60-40 \%$ relation of NoteDocument, being Note objects the most accessed ones $(60 \%)$. Consequently, the meaning of the rest of indicators has a low significance. As a result, these data offer a clear picture both of the way group activity tends to be distributed and the way the CSCL tool is used. Figure 4 recomposes the results of table 1 and shows the tendencies of users activity distribution in a more graphical and concise way.

In an effort to identify patterns of collaboration, we

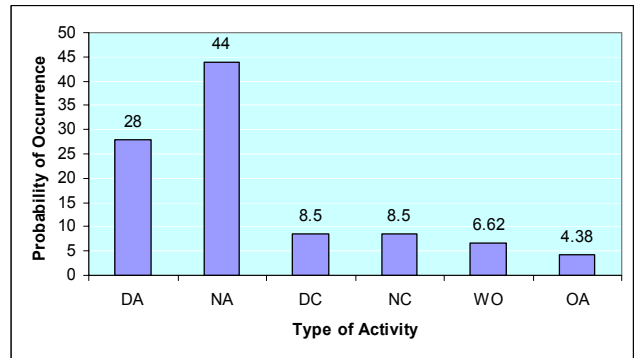

Figure 4. Distribution of the users' activity that shows the participation-behavior during collaboration. Where DA: Doc Access, NA: Note Access, DC: Doc Creation, NC: Note Creation, WO: Workspace Organization, OA: Other Activities.

organize group interaction in six main activity categories: document creation and access, note creation and access, workspace organization and other activities, which are measured by probabilities of occurrence. In our case, workspace organization is composed by the accumulated values specific events such as RateEvent, DropEvent, ChangeDescEvent, RenameEvent, CutEvent, ReplaceEvent and
DeleteEvent. The results of our analysis show that the probability of occurrence of each of the above activity categories is the following: for a document access (DA) is $28 \%$ (which results from multiplying the ReadEvent indicator 0.74 by the doc access level 0.39 ), for a note access (NA) is $44 \%$ (multiplying the ReadEvent indicator 0.74 by the note access level $0.59)$. The probability of occurrence of the rest of the actions is significantly lower, being an $8.5 \%$ for doc and note creation (DC, NC), resulting from multiplying the CreateEvent indicator 0.18 by the note/doc access level 0.47 , and a $6.62 \%$ for workspace organization (WO), which is the accumulated values of organization events; finally, all the rest of available actions (OA) occur with a $4.38 \%$ probability.

The Knowledge Server is capable of giving detailed

Table 1. Different details of global activity discovered using the knowledge server.

\begin{tabular}{|c|c|}
\hline \multicolumn{2}{|c|}{ Global Activity Distribution } \\
\hline Total \# of Events & 77157 \\
\hline Avg Events/User & 221.716 \\
\hline \multicolumn{2}{|c|}{ Global $\%$ of Events/Category } \\
\hline Event Category & $\%$ \\
\hline BranchEvent & 0.005 \\
\hline ChangeDateEvent & 0.01 \\
\hline ChangeDescEvent & 0.818 \\
\hline ChangeEvent & 0.086 \\
\hline CheckinEvent & 0.021 \\
\hline CheckoutEvent & 0.022 \\
\hline ChvinfoEvent & 0.009 \\
\hline CopyEvent & 0 \\
\hline CreateEvent & 18.079 \\
\hline CutEvent & 0.999 \\
\hline DeleteEvent & 1.581 \\
\hline DropEvent & 0.708 \\
\hline EditEvent & 0.614 \\
\hline ForgetEvent & 0.001 \\
\hline LinkEvent & 0.118 \\
\hline RateEvent & 0.417 \\
\hline ReadEvent & 74.001 \\
\hline RenameEvent & 0.903 \\
\hline ReplaceEvent & 1.196 \\
\hline ReviseEvent & 0.248 \\
\hline UndeleteEvent & 0.03 \\
\hline VersionEvent & 0.133 \\
\hline
\end{tabular}

\begin{tabular}{|c|c|}
\hline Total of Objects Created & 13949 \\
\hline$\%$ of Creative Activity & 18.079 \\
\hline $\begin{array}{ll}\text { Avg } & \text { Objects } \\
\text { Created/User } & \end{array}$ & 40.083 \\
\hline \multicolumn{2}{|c|}{ Global $\%$ of Object/Type } \\
\hline \multicolumn{2}{|c|}{ Object Type $\%$} \\
\hline Appointment & 0.652 \\
\hline Document & 46.756 \\
\hline Folder & 3.843 \\
\hline Note & 47.394 \\
\hline Noteboard & 0 \\
\hline Notes & 1.341 \\
\hline WSCalendar & 0.014 \\
\hline $\begin{array}{l}\text { Total of Objects } \\
\text { Accessed }\end{array}$ & 63208 \\
\hline$\%$ of Perceptive Activity & 81.921 \\
\hline $\begin{array}{c}\text { Avg Objects } \\
\text { Accessed/User }\end{array}$ & 181.632 \\
\hline \multicolumn{2}{|c|}{ Global \% of Object/Type } \\
\hline Object Type & $\%$ \\
\hline Appointment & 0.196 \\
\hline Document & 38.963 \\
\hline Folder & 1.321 \\
\hline Note & 59.005 \\
\hline Noteboard & 0 \\
\hline Notes & 0.511 \\
\hline WSCalendar & 0.003 \\
\hline
\end{tabular}

information about group and individual activity, based on the awareness knowledge that is collected by the complex social network which models the whole collaborative learning activity. It is possible to analyze activity details for every participant and every workgroup in an efficient and fairly rapid manner. Table 2 shows an example that presents more refined information about the activity of a specific user, which is extracted from the Knowledge Server.

Comparing the activity information of the individual user shown in table 2 with the general pattern of collaborative activity identified in table 1 , the evaluator of the collaborative activity can discern that this user has created a number of documents $(68,3 \%)$ which is far above the average of the global document creation $(46,7 \%)$. Thus, the distribution of 
document and note creation of the user does not follow the balanced $50-50 \%$ global tendency of the general pattern; instead, the user gives more importance to document rather than note creation. The evaluator will then have to figure out the quality of user's documents as well the user's true intentions underlying his/her notes through a qualitative assessment. In this sense, the conversation analysis approach we are currently developing will support the evaluator's decision making process more efficiently. Moreover, the user's document and note access activity does not follow the general $40-60 \%$ pattern; in fact, it is more balanced, being closed to $50-50 \%$. In general, in an effort to connect and interpret these results according to the proposed ontology (in Figure 3), we can say that the user's creative activity $(24,4 \%)$, which forms part of his/her active learning behavior, is above the average global one (18\%), whereas the user's perceptive attitude seems more balanced, though he/she shows a somewhat lower reading activity $(69,6 \%)$ as opposed to the $74 \%$ of the general pattern. Making this comparison, the evaluator is able to analyze specific details of every user's activity as well as to measure the user's performance in terms of the general expected behavior of the whole class.

As regards the analysis of learning group performance, the Knowledge Server is able to extract precise details for the activity of a specific group and build a detailed report for the social network. Table 3 shows the report generated for a given group.

Table 2. Detailed information activity for a specific user,

\begin{tabular}{|c|l|}
\hline \multicolumn{2}{|c|}{ extracted from the Knowledge Server. } \\
Astivity review for participant: & $\begin{array}{l}\text { * Total of Events (168) } \\
\text { Erequency of Events Performed: } \\
\text { BranchEvent (0): } 0 \%\end{array}$ \\
* Created Objects (41) & ChangeDateEvent (0): $0 \%$ \\
Frequency of Created Objects Types: & ChangeDescEvent (0): $0 \%$ \\
Appointment (1): $2.44 \%$ & ChangeEvent (0): $0 \%$ \\
Document (28): $68.29 \%$ & CheckinEvent (0): $0 \%$ \\
Folder (0): $0 \%$ & CheckoutEvent (0): $0 \%$ \\
Note (10): $24.39 \%$ & ChvinfoEvent (0): $0 \%$ \\
Noteboard (0): $0 \%$ & CopyEvent (0): $0 \%$ \\
Notes (2): $4.88 \%$ & CreateEvent (41): $24.4 \%$ \\
WSCalendar (0): $0 \%$ & CutEvent (0): $0 \%$ \\
& DeleteEvent (9): $5.36 \%$ \\
* Total \# of Accesses (127) & DropEvent (0): $0 \%$ \\
Average Time spent in object access (2.52 & EditEvent (0): $0 \%$ \\
hours) & ForgetEvent (0): $0 \%$ \\
Frequency of Object Types Accessed: & LinkEvent (1): $0.6 \%$ \\
Appointment (1): $0.79 \%$ & RateEvent (0): $0 \%$ \\
Document (65): $51.18 \%$ & ReadEvent (117): $69.64 \%$ \\
Folder (0): $0 \%$ & RenameEvent (0): $0 \%$ \\
Note (59): $46.46 \%$ & ReplaceEvent (0): $0 \%$ \\
Noteboard (0): $0 \%$ & ReviseEvent (0): $0 \%$ \\
Notes (2): $1.57 \%$ & UndeleteEvent (0): $0 \%$ \\
WSCalendar (0): $0 \%$ & VersionEvent (0): $0 \%$ \\
\hline
\end{tabular}

The information available for a learning group goes beyond the one available for a single user. In particular, the Knowledge Server provides detailed information about the way activity is distributed, including information about the members' creative and perceptive behavior. Besides, there are plenty of details regarding the interaction volume produced by every pair of members when they access common objects; for instance, the interaction "User-2695683 => User-2697485: 183" means that User-2695683 accessed the objects created by User-2697485 183 times. In addition, we also count the number of accesses that every member produces outside their group. As in the case of individual performance analysis, group performance analysis can be also compared to the performance shown by the total number of collaborative groups. Finally, our system can also extract information related to the social network formed by a group that includes connection weights for the creative and perceptive collaborative categories. This is done by relating the Internal Interaction values with the Activity Distribution ones, as shown in table 3. For instance, the User-2695683 created 197 knowledge objects which were accessed 367 times by the other group partners (averaging 122.3 times); this means that $62.08 \%$ of his creation activity was accessed by partners.

Table 3. Report for a specific group produced by the Knowledge Server.

\begin{tabular}{|c|c|}
\hline \\
\hline G: Group-2757651 & *** Activity Distribution $* * *$ \\
\hline Members: & * Creative Activity per Member * \\
\hline User-2695683 & User-2695683(197): 36.08\% \\
\hline User-2697485 & User-2697485(192): $35.16 \%$ \\
\hline User-2697789 & User-2697789(98): 17.95\% \\
\hline User-2749058 & User-2749058(59): 10.81\% \\
\hline * Objects Created by Group (546) & * Perceptive Activity per Member * \\
\hline Average \# of Objects per Member (136.5) & User-2695683(686): $31.98 \%$ \\
\hline Frequency of Object Types Created: & User-2697485(658): $30.68 \%$ \\
\hline Appointment (8): $1.47 \%$ & User-2697789(367): $17.11 \%$ \\
\hline Document (252): $46.15 \%$ & User-2749058(434): $20.23 \%$ \\
\hline Folder (120): $21.98 \%$ & \\
\hline Note (680): $124.54 \%$ & $* * *$ INTERACTION ACTIVITY $* * *$ \\
\hline Noteboard (0): $0 \%$ & * Internal Interaction * \\
\hline Notes (32): $5.86 \%$ & $m b r=>m b r: \#$ intercts. through objs \\
\hline WSCalendar (0): $0 \%$ & \\
\hline * Total \# of Events of Group (5382) & 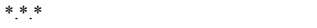 \\
\hline Average \# of Events per Member (1345.5) & (a) User-2695683 $\Rightarrow$ User-2695683: \\
\hline Frequency of Events Performed: & \\
\hline BranchEvent $(0): 0 \%$ & User-2695683 => User-2697485: 183 \\
\hline ChangeDateEvent $(0): 0 \%$ & User-2695683 => User-2697789: 66 \\
\hline ChangeDescEvent (206): $0.83 \%$ & User-2695683 $\Rightarrow$ User-2749058: 40 \\
\hline ChangeEvent (18): $0.33 \%$ & *.*.* \\
\hline CheckinEvent (0): $0 \%$ & User-2697485 => User-2695683: 142 \\
\hline CheckoutEvent (0): $0 \%$ & (a) User-2697485 $\Rightarrow$ User-2697485: \\
\hline ChvinfoEvent (0): $0 \%$ & 430 \\
\hline CopyEvent (0): $0 \%$ & User-2697485 $\Rightarrow>$ User-2697789: 43 \\
\hline CreateEvent (1092): $20.29 \%$ & User-2697485 => User-2749058: 29 \\
\hline CutEvent (76): $1.41 \%$ & 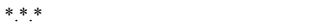 \\
\hline DeleteEvent (122): $2.27 \%$ & User-2697789 => User-2695683: 95 \\
\hline DropEvent (62): $1.15 \%$ & User-2697789 => User-2697485: 91 \\
\hline EditEvent (70): $1.3 \%$ & (a) User-2697789 $\Rightarrow$ User-2697789: \\
\hline ForgetEvent (0): $0 \%$ & 150 \\
\hline LinkEvent (8): $0.15 \%$ & User-2697789 => User-2749058: 24 \\
\hline RateEvent (2): $0.04 \%$ & 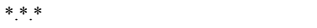 \\
\hline ReadEvent (3350): 62.24\% & User-2749058 $\Rightarrow$ User-2695683: 130 \\
\hline RenameEvent (60): $1.11 \%$ & User-2749058 $\Rightarrow$ User-2697485: 133 \\
\hline ReplaceEvent (312): $5.8 \%$ & User-2749058 $\Rightarrow$ User-2697789: 60 \\
\hline ReviseEvent (0): $0 \%$ & (a) User-2749058 => User-2749058: 85 \\
\hline UndeleteEvent (4): $0.07 \%$ & 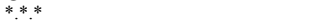 \\
\hline VersionEvent (0): $0 \%$ & \\
\hline * Total \# of Accesses of Group (2145) & $*$ External Interaction * \\
\hline Average \# of Accesses per Member (536.25) & User-2695683: 56 accesses outside \\
\hline Average Time spent in obj. access ( 2.5 hours) & User-2697485: 14 accesses outside \\
\hline Frequency of Object Types Acceded: & User-2697789: 7 accesses outside \\
\hline Appointment (8): $0.37 \%$ & User-2749058: 26 accesses outside \\
\hline Document (1664): $77.58 \%$ & \\
\hline Folder (262): $12.21 \%$ & \\
\hline Note (2280): $10.62 \%$ & \\
\hline Noteboard (0): $0 \%$ & \\
\hline Notes (76): $3.54 \%$ & \\
\hline WSCalendar (0): $0 \%$ & \\
\hline
\end{tabular}




\section{Conclusions and future work}

This paper presents an approach for analyzing online collaborative activity by integrating different strategies for coping with a variety of issues of the problem, mainly a quantitative and a qualitative technique.

The analysis model has been conceptualized in a layered manner that resulted from the definition of generic to more specific activity indicators that were specified through an ontological approach. This paper focuses on the quantitative method of analysis which was based on social network analysis (SNA) and consists in building a network using the information stored in log-files. The social network is the axis of a Knowledge Server which is capable of answering to different queries involving performance, interaction and collaboration for distributed problem solving.

Temporal logics were defined to measure collaborative actions, whereas the ontology represents and classifies the primitive actions performed by participants. By setting order to primitive events and providing semantics to specific sequences of primitive actions, collaboration analysis acquires a formal basement. The social network organization satisfies and supports the ontological approach, by explaining and feeding the different awareness requirements.

Currently, the Knowledge Server has more awareness information of the collaborative activity than needed. Nevertheless, this awareness information will be needed to feed the inference mechanisms when the qualitative stage will be built.

Finally, two search agents were built to improve the performance of the Knowledge Server. These agents are able to work in different computers as in a distributed fashion. The use of a more complete agentbased approach will be more evident during the qualitative stage, in which more specific agents will be employed to support the performance of complex tasks, such as a conversation analysis. Content analysis of the conversation will substantially complement the quantitative process. In particular, the results obtained from the social network quantitative analysis will be completed by the identification of the participants' intentions underlying their contributions in a conversation.

Based on such an integrated approach and the further enrichment of the ontology presented in this paper, future work aims at the full development of a solution for the analysis of online collaboration.

Acknowledgements. This work has been partially supported by the Spanish MCYT project TSI2005-08225-C07-05.

\section{References}

[1] Carroll, J. M., Rosson, M. B., Convertino, G., Ganoe, C.: $\mathrm{H}$. Awareness and teamwork in computer-supported collaborations. Interacting with Computers, volume 18, pp 21-46. Elsevier Science Ltd. (2006).

[2] Daradoumis T., Martínez A., and Xhafa, F.: A Layered Framework for Evaluating Online Collaborative Learning Interactions. International Journal of Human-Computer Studies. Special Issue on "Theoretical and Empirical Advances in Groupware Research", Academic Press: Elsevier Ltd., ISSN: 1071-5819, Volume 64, Issue 7, July 2006, pp. 622-635.

[3] Daradoumis, T. and Casillas, L.A.: A Neural Approach for Modeling the Inference of Awareness in ComputerSupported Collaboration. Lecture Notes in Computer Science (LNCS 4227). Innovative Approaches for Learning and Knowledge Sharing. W. Nejdl and K. Tochtermann (Eds.). Springer-Verlag Berlin Heidelberg, ISBN: 3-540-45777-1, pp. $464-469$. (2006).

[4] Endsley, M.: Toward a theory of situation awareness in dynamic systems. Human factors, Volume 37 Issue 1. ISSN 0018-7208. Texas, USA. (1995).

[5] Fisher, M.: Implementing Temporal Logics: Tools for Execution and Proof. Proceedings of the Sixth International Workshop on Computational Logic in Multi-Agent System (CLIMA); Lecture Notes in Artificial Intelligence 3900, Springer-Verlag; London, U.K. (2006).

[6] Soller, A.: Supporting Social Interaction in an Intelligent Collaborative Learning System. International Journal of Artificial Intelligence in Education, hosted by the School of Informatics at The University of Edinburgh; Edinburgh, U.K. (2001).

[7] Soller, A.: Computational Modeling and Analysis of Knowledge Sharing in Collaborative Distance Learning. User Modeling and User-Adapted Interaction, number 14; Kluwer Academic Publishers. Printed in the Netherlands. (2004).

[8] Soller, A., Lesgold, A.: Analyzing Peer Dialogue from an Active Learning Perspective. Proceedings of the AI-ED 99 Workshop: Analysing Educational Dialogue Interaction: Towards Models that Support Learning, LeMans, France. (1999).

[9] Soller, A., Lesgold, A.: Modeling the Process of Collaborative Learning. International Workshop on New Technologies in Collaborative Learning, Awaji-Yumebutai, Japan. (2000). 